\title{
Transdermal Lidocaine and Ketamine for Neuropathic Pain: A Study of Effectiveness and Tolerability
}

\author{
Emily Tam and Andrea D. Furlan* \\ Toronto Rehabilitation Institute, (University Health Network) 550 University Av, room 12-020, Toronto, ON, M5G 2A2, \\ Canada
}

Abstr act: Background: Acute neuropathic pain is a common disorder. Transdermal cream could be an alternative to oral medications.

Objective: To evaluate the effectiveness and tolerability of transdermal Lidocaine and Ketamine for acute neuropathic pain.

Study Design: Retrospective chart review

Setting: University-affiliated outpatient Physiatry clinic

Methods: articipants: neuropathic pain with a prescription of a transdermal cream containing Lidocaine and Ketamine. Effectiveness was evaluated by the number of patients with improvement divided by the total number of patients who received a prescription of the cream.

Results: A total of 854 patient charts were reviewed. Twenty-one patients with symptoms, signs, and/or a documented diagnosis of neuropathic pain and had been given a prescription of a transdermal preparation containing Lidocaine and Ketamine. Four groups were identified: those with a clearly stated diagnosis of neuropathic pain and prescribed a transdermal compound containing Lidocaine and Ketamine with follow-up (Group A) or without follow-up (Group B), and those with a suggested diagnosis of neuropathic pain with (Group C) or without follow-up (Group D). Effectiveness of the cream was seven out of eight (87\%) for Group A and one out of three (33\%) for Group C. In total, eight out of 11 patients (73\%) benefited from a cream containing Lidocaine and Ketamine. Two patients experienced skin reactions that led to discontinuation of treatment.

Limitations: This is a retrospective chart review without control group.

Conclusion: Transdermal cream containing Ketamine and Lidocaine was effective in $73 \%$ of patients with acute neuropathic pain and may be a good alternative to oral medications.

Keywor ds: Transdermal, ketamine, lidocaine, neuropathic pain.

\section{INTRODUCTION}

Neuropathic pain $(\mathrm{NeP})$, as defined by the International Association for the Study of Pain (IASP), is a result of a primary lesion or dysfunction of the nervous system. NeP, therefore, is a possible outcome from a wide variety of central and peripheral nerve disorders. An estimate of the prevalence of chronic $\mathrm{NeP}$ in the general population is $8.2 \%$ [1]. The Canadian Pain Society (CPS) has suggested that up to one million Canadians may have NeP [2]. And the tendency is the prevalence of $\mathrm{NeP}$ will increase with the aging of population, because several $\mathrm{NeP}$ syndromes such as painful diabetic neuropathy are more common in the elderly.

Clinical features of NeP include allodynia, hyperalgesia, numbness, weakness, and spontaneous pain that is burning, shooting, or shock-like. Symptoms are usually quite severe

*Address correspondence to this author at the Toronto Rehabilitation Institute, 550 University Av, room 12-020, Toronto, ON, M5G 2A2, Canada;

Tel: 416597 3422; Ext: 4607; Fax: 4165166373;

E-mail: Andrea.Furlan@uhn.ca and may become chronic. In addition, NeP impairs participants' mood, quality of life, activities of daily living, and performance at work [3].

The pathophysiology of $\mathrm{NeP}$ is incompletely understood, however, many mechanisms have been put forth in the literature. The pain pathway involves the peripheral nerves, spinal cord, and the brain. NeP can develop from dysfunction at any one or more of these levels [4].

At the peripheral level, hypersensitivity is postulated to be secondary to post-nerve injury and inflammation results in the sensitization of nociceptors through alteration of sodium channel distribution and function [5]. Abnormally expressed sodium channels in $\mathrm{C}$ fibre nociceptors result in spontaneous ectopic activity [6]. Other receptors and release of neurotransmitters, such as substance $P$, are also altered post-inflammation. In addition, neuromas form on adjacent, uninjured nerves, leading to mechanosensitivity and expanded receptive fields. Gene transcription is altered in dorsal root ganglia, sodium channels are upregulated and calcium channels are altered to produce allodynia [6]. 
At the central level, sensitization of dorsal horn neurons can be induced by amplified and facilitated activity from peripheral nociceptors [7]. For example, molecular changes in central neurons enhance the response in pain transmission neurons [8]. In addition, descending pathways are 'disinhibited' by lower levels of inhibitory transmitters, such as GABA and glycine, in spinal cord dorsal horn neurons. Thirdly, activation of N-methyl-D-aspartate (NMDA) receptors lowers the threshold for nerve transduction, as well as facilitates synaptic transmissions.[9] NMDA receptors are involved in sensory input at the level of the spinal cord, thalamus, limbic system, and cerebral cortex.

The underlying causes of NeP may also be divided into central and peripheral causes. Central neuropathic pain includes poststroke pain, below-level pain after spinal cord injury, pain in multiple sclerosis and phantom limb pain. Peripheral mononeuropathies include postherpetic neuralgia, radiculopathy, and carpal tunnel syndrome. Polyneuropathies include diabetic and HIV neuropathies. Complex regional pain syndrome is also an example of $\mathrm{NeP}$.

Development of effective therapies is in its infancy. Nonpharmacologic management includes: exercise, transcutaneous electrical nerve stimulation (TENS), percutaneous electrical nerve stimulation (PENS), graded motor imagery, cognitive behavioural therapy, and supportive psychotherapy. Little evidence for these management options has been published in the literature [3].

The Canadian Pain Society published evidence-based guidelines for the pharmacologic management of NeP.[2] The International Association for the Study of Pain also published evidence-based recommendations for the pharmacologic management of $\mathrm{NeP}$ : first-line recommendations are tricyclic antidepressants, gabapentin and pregabalin, and topical lidocaine, while opioid analgesics, tramadol, certain antiepileptics, and topical capsaicin are chosen based on individual patient tolerability and comorbidities [10]. The success, however, of oral medications in $\mathrm{NeP}$ has been limited by systemic side effects.

Systemic adverse effects, therefore, are problematic when pharmacologically managing $\mathrm{NeP}$ via the oral route. One alternative is local delivery, a method that bypasses first-pass metabolism in the liver and therefore, decreases dosage requirements [11]. The topical drug delivery route, for instance, aims to provide direct relief to the area of pain. Few studies in the literature investigate topical therapies for $\mathrm{NeP}$. A randomized, double-blind, placebo-controlled study involving 151 participants found that topical doxepin (TCA), capsaicin, and a combination of both, produce a similar degree of analgesia, but onset of analgesia is faster with the combination topical [12].

To date, evidence-based recommendations for topical analgesia for $\mathrm{NeP}$ remains mixed. An alternative to local topical analgesia is transdermal drug delivery, a method that also eliminates the difficulties associated with oral medications with poor or variable gastrointestinal absorption. Whereas topical medications allow for isolated peripheral drug activity, transdermal medications can act locally and are also absorbed systemically. Systemic absorption is important to target central sensitization mechanisms in NeP. The bene- fits of transdermals include steady-state delivery of drug, with reduced peak plasma drug concentrations that can reduce dosage-related adverse effects [11]. increased compliance, and high-concentration local drug delivery with reduced systemic toxicity.

Transdermal drug delivery requires compounds of low molecular weight and lipophilicity to enhance penetration through the stratum corneum of the skin. A commonly used transdermal drug delivery vehicle is pluronic lecithin organogel (PLO). PLO is composed of isopropyl palmitate, soy lecithin, water, and Pluronic F127; the first two components form the oil phase and the latter two, the aqueous phase [13].

With the current understanding of the pathophysiology of $\mathrm{NeP}$, we propose that pain management will be more effective if targeted transdermally at both the peripheral and central levels. Lidocaine is an anesthetic that dampens peripheral nociceptive sensitization by inhibiting neural ectopic discharges via sodium channel blockade, thereby also altering signals upstream and centrally. Ketamine is an NMDA receptor antagonist that decreases central sensitization and modulation by lowering the threshold for nerve transduction and reduces the effects of substance P. Ketamine also targets the opioid receptor, as well as sodium and potassium channels, to reduce pain. Additionally, it may alter the docking station for vesicles containing neurotransmitters, including glutamate. We hypothesize that a transdermal preparation of lidocaine and ketamine, targeting both peripheral and central mechanisms of NeP, will be effective for the management of acute $\mathrm{NeP}$.

\section{MATERIALS AND METHODS}

The charts of patients who attended the General Physiatry Outpatient Clinics of three Physiatrists at a rehabilitation hospital in Toronto, ON (Toronto Rehabilitation Institute, Hillcrest Centre) between May 30, 2007 and June 1, 2009 were manually reviewed to identify patients 18 years of age and older with a diagnosis of neuropathic pain as a result of any condition and had received a prescription of a transdermal preparation containing Lidocaine and Ketamine. An electronic search of electronic clinic notes was performed to ensure potential participants were not missed during the manual chart review.

Patient age, sex and primary diagnoses relating to neuropathic pain were noted. Patients who had had prior treatment with any transdermal preparations were excluded. All coadministrations of medications for pain were documented. Transdermal preparations containing other substances other than Lidocaine and Ketamine were noted. The percentage of Lidocaine and Ketamine, and other substances in the transdermal preparation were recorded. The frequency and location of application of the transdermal preparation were noted. Adverse reaction(s) and reasons for discontinuing use of the transdermal preparation were recorded. The study protocol was approved by the hospital ethics review board.

Descriptive data synthesis and quantitative data analysis were performed. The effectiveness of the transdermal preparation was evaluated by the number of patients with improvement (n) compared to the total number of patients who 
received a prescription of the transdermal preparation $(\mathrm{N})$. Improvement was evaluated by meaningful pain relief. Definitions of meaningful pain relief have been previously described in a study by Sandoval et al. [14]. The definitions from this study have been adapted for the purposes of this study as follows:

"Meaningful" relief was defined when at least 1 of the following was found:

A. Significant changes in any of multiple quantitatively measured outcomes, for example, VAS score, specific questionnaire, or percentage of pain relief;

B. "Satisfactory" or "acceptable" pain relief as deemed by the patients or physicians;

C. "Worthwhile", relief obtained through narratives; for example, pain relief more than 30\%, concurrent improvement on various dimensions (pain, function, fewer side effects, return to work, etc.).

“'Nonmeaningful'” relief was defined when at least 1 of the following occurred:

A. Relief less than $30 \%$ of pain reduction (this percentage was arbitrarily agreed upon by the authors of this present study);
B. "Mild" or "no" relief of the original pain, as deemed by the patients or physicians.

Frequencies of adverse effects were reported as absolute numbers and percentages.

\section{RESULTS}

A total of 854 patient charts were reviewed. Twenty-one patients with symptoms, signs, and/or a documented diagnosis of neuropathic pain and had been given a prescription of a transdermal preparation containing Lidocaine and Ketamine were identified. The age range of the patients was 22 to 72 years of age (Fig. 1). The median age was 47 . Nine females and 12 males were included in the study. Past medical histories of all patients were remarkable only for diagnoses related to neuropathic pain. A range of underlying diagnoses contributing to neuropathic symptoms was found (Fig. 2). The most frequent diagnosis was trauma (43\%). A number of medications, taken orally, were used for pain, the most frequent being opioids (Fig. 3). Nerve membrane stabilizers and tricyclic antidepressants were also frequently prescribed.

The transdermal combination of Lidocaine/Ketamine/ Diclofenac was prescribed to 6 patients (29\%) (Fig. 4). The exclusive combination of Lidocaine and Ketamine was pre-

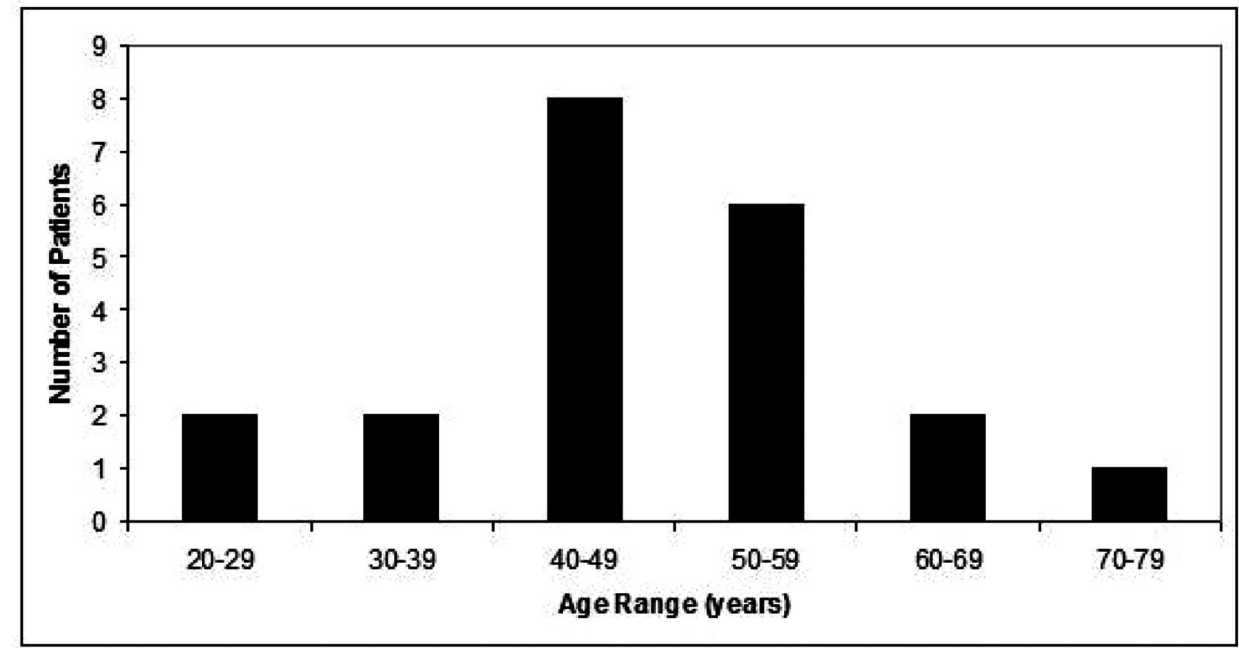

Fig. (1). Age range of patients.

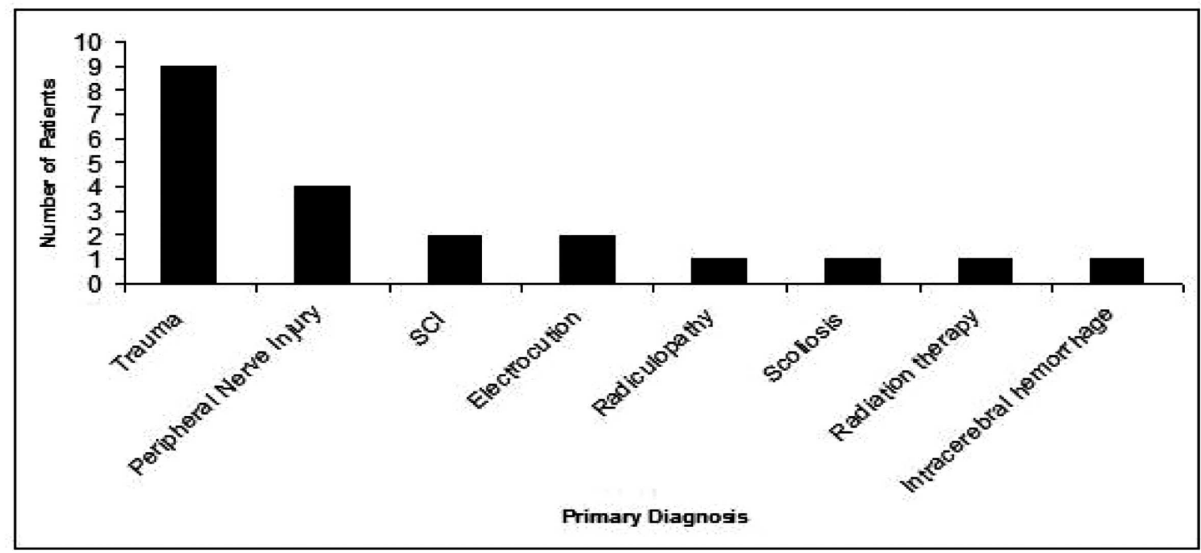

Fig. (2). Underlying diagnoses contributing to neuropathic symptoms. 


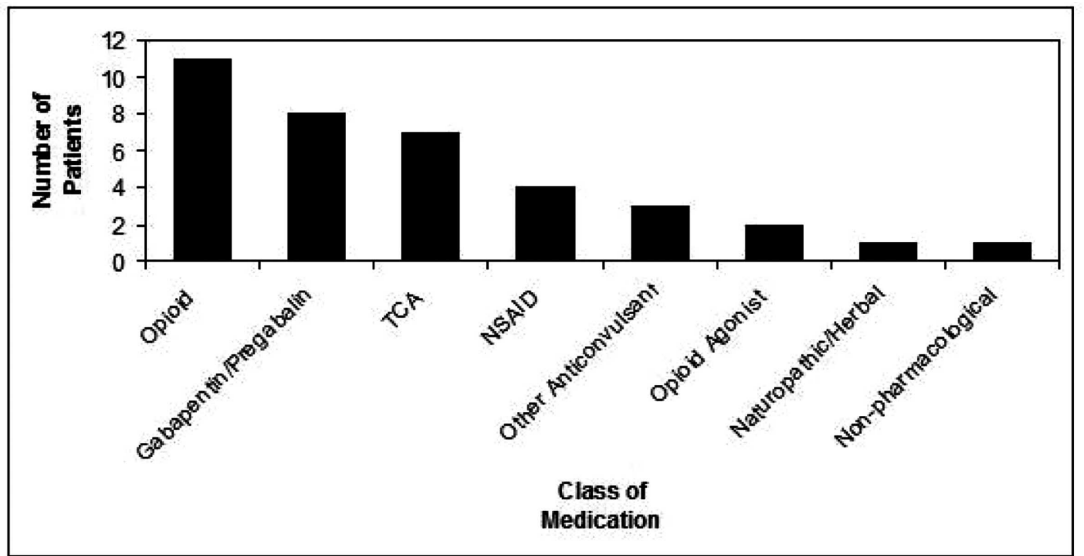

Fig. (3). Concomitant Use of Medications for Neuropathic Pain.

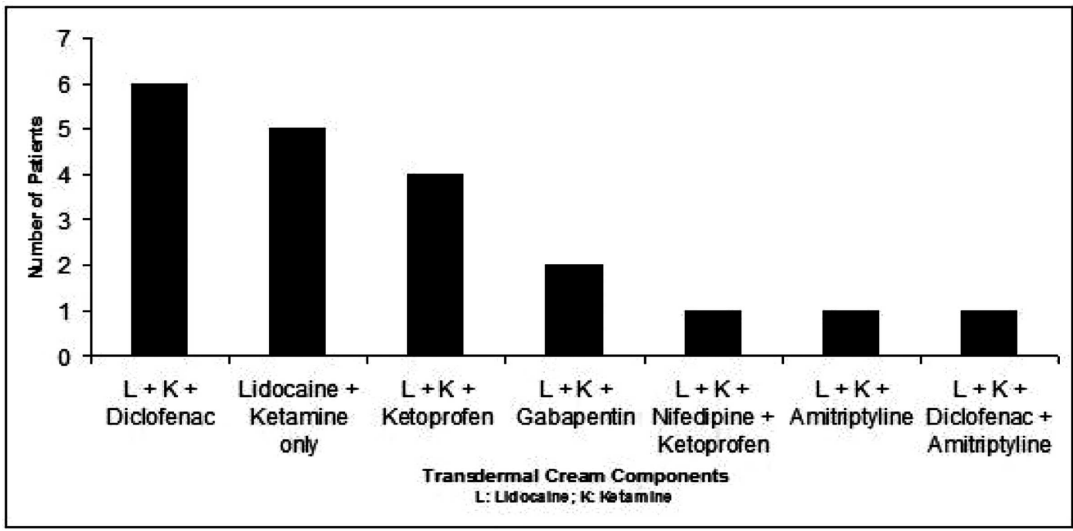

Fig. (4). Components of Transdermal Creams Prescribed.

scribed in 5 of the patients (24\%). Other medications that were compounded with Lidocaine and Ketamine were Ketoprofen, Gabapentin, Amitriptyline, and Nifedipine.

Various percentages of each compound were prescribed. The range prescribed for Lidocaine and Ketamine, respectively, was $2-10 \%$ and $5-10 \%$. The base in which these transdermal medications were compounded also varied. PLO was the most frequently prescribed (52\%). Lipoderm was also used as a compounding base (19\%); the remainder of patient charts did not have documentation of a compounding base. For the 16 charts that contained information about the frequency of application of the transdermal preparation, 14 indicated TID, and 2 indicated TID prn.

The ankle and foot were the most frequently affected body parts, as indicated in the prescriptions. (Fig. 5) Neuropathic symptoms most frequently affected the extremities (arm, hand, wrist, leg, knee, ankle, foot). Fewer prescriptions were written for the axial body in this set of patients.

Not all patient charts contained a documented diagnosis of neuropathic pain, not all had documentation of the transdermal prescription, and not all had documentation of follow-up of the neuropathic pain symptoms and/or transdermal preparation. To determine the effectiveness of the transdermal, however, documented follow-up was required. These 21 patients, therefore, were divided into 4 groups based on presence of a formal documented diagnosis of neuropathic pain and presence of follow-up regarding the effect of the transdermal preparation.
Patients in Group A, n=8, had a documented diagnosis of neuropathic pain and had follow-up describing the effect of the transdermal preparation. Those in Group B, n=3, had a documented diagnosis, but no follow-up. Patients in Group $\mathrm{C}, \mathrm{n}=3$, had a suggested diagnosis of neuropathic pain (based on the history and physical described in the clinic note) and follow-up. A suggested diagnosis was determined by the presence of a primary diagnosis that could be related to neuropathic pain and the presence of terms such as, "burning", "numbness”, “shooting pain”, “allodynia”, “quasineuropathic features", and "hyperpathia", in addition to a concurrent recommendation in the clinic note for a transdermal preparation contaning Lidocaine and Ketamine. Those in Group D, $n=6$, had a suggested diagnosis of neuropathic pain and no follow-up.

Seven of 8 patients in Group A had a meaningful response to the transdermal cream; the effectiveness of the transdermal cream was $88 \%$ for Group A. One of 3 Group C patients had a meaningful response the effectiveness was 33\% for Group C. The overall effectiveness of the transdermal cream in this study was, therefore, 73\%.

Five of the eight patients in Group A used a transdermal preparation that contained exclusively Lidocaine and Ketamine; the percentages of each of these components that were prescribed varied from patient to patient. Of these five, one did not find the Lidocaine/Ketamine combination effective. None of the patients in Group C used transdermals that only contained Lidocaine and Ketamine. 


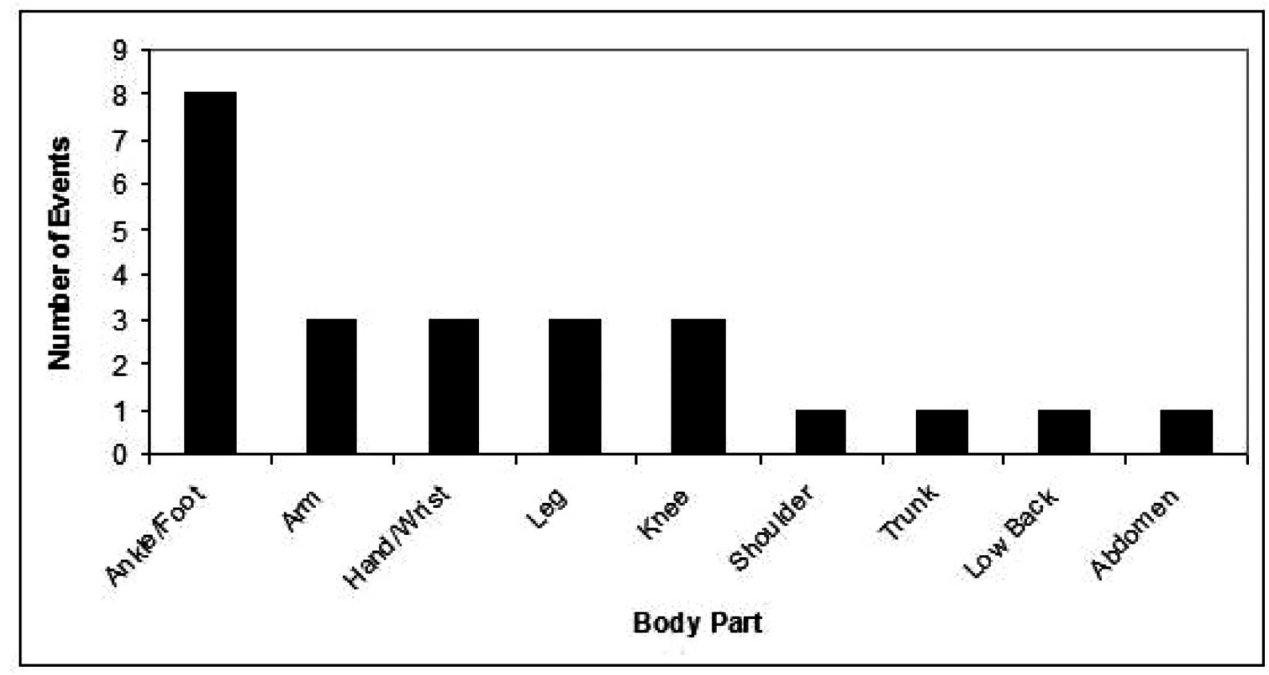

Fig. (5). Body Parts Affected by Neuropathic Pain.

Two patients in Group A experienced adverse skin reactions that led to discontinuation of the transdermal cream. These reactions were documented as "hives" and "rash/dermatitis". The frequency of adverse effects in patients who had follow-up was 18\% (2/11). Overall, the frequency of adverse effects in this study was $10 \%$. The patient who developed hives had applied a transdermal containing 2\% Lidocaine/5\% Ketamine/10\% Diclofenac in a PLO base. It was discontinued and a transdermal containing $2 \%$ Lidocaine/10\% Diclofenac in Lipoderm base was prescribed without dermatitis and the patient "didn't notice it to be as effective". The patient who developed dermatitis had applied a transdermal containing $10 \%$ Lidocaine $/ 10 \%$ Ketamine (base unknown). It was discontinued and the patient was given a transdermal containing $10 \%$ Lidocaine $/ 5 \%$ Diclofenac/5\% Amitriptyline (base unknown) that also produced dermatitis. A subsequent clinic note states continued use of a Lidocaine/Ketamine transdermal preparation (base unknown), but no prescription was found in the chart, and no further follow-up was documented. It was not clear in the clinic notes if other patients in Groups A or C discontinued the transdermal.

\section{DISCUSSION}

This study sought to characterize the effectiveness of a transdermal preparation of Lidocaine and Ketamine for the management of acute NeP by targeting both peripheral and central mechanisms of NeP. Previous studies have examined both topical Ketamine and topical Lidocaine preparations, either in combination with other analgesics, or alone.

Topical Ketamine for the management of $\mathrm{NeP}$ has been investigated in one study in the literature. Topical amitriptyline (2\%) and ketamine (1\%) for $\mathrm{NeP}$ were investigated in 92 participants enrolled in a randomized, double-blind, placebocontrolled study [15]. A decrease in pain scores of 1 to 1.5 units was seen between test groups of amitriptyline, ketamine, and a combination of the two. No significant differences in pain scores were found between the groups; however, optimal doses were not established in this study to definitively conclude the effectiveness of each study group.
Two studies examine Topical Lidocaine in the management of NeP. Ho et al. [16] conducted a randomized, placebo-controlled crossover study to evaluate the efficacy of topical 5\% amitriptyline and 5\% lidocaine in the treatment of $\mathrm{NeP}$. The lidocaine group had significant reductions in the visual analog scale, but had minimal clinical improvement. No significant changes were observed in the amitriptyline and placebo groups.

The only topical analgesic recommended in the CPS 2007 guidelines was the 5\% lidocaine patch. Meier et al. [17] conducted a randomized, double-blind, placebocontrolled study $(n=40)$ and found that a $5 \%$ lidocaine patch, as an add-on therapy, is clearly effective in reducing ongoing neuropathic pain $(\mathrm{p}=0.017)$ and allodynia $(\mathrm{p}=$ 0.023 ) in the first 8 hours of treatment. The patch was also effective in reducing symptoms over 7 days $(\mathrm{p}=0.018)$. The NNT was 4.4 to achieve $>50 \%$ relief of ongoing pain. A Cochrane Review [18] of 3 trials evaluated topical lidocaine in the treatment of postherpetic neuralgia. A meta-analysis of two trials that reported pain relief (the third reported on secondary outcome measures) found a statistical difference between topical lidocaine and control groups in the relief of postherpetic neuralgia $(p=0.003)$. Evidence to support topical lidocaine as a first-line agent in the treatment of postherpetic neuralgia was, however, insufficient.

In this study, a chart review was performed to identify patients with a diagnosis of $\mathrm{NeP}$ who received a prescription of a transdermal preparation containing Lidocaine and Ketamine. For those patients who had a definitive and documented diagnosis of neuropathic pain and follow-up, the transdermal preparation containing Lidocaine and Ketamine was effective; $88 \%$ found using the transdermal resulted in meaningful relief. Overall, the effectiveness of the transdermal cream in this study was $73 \%$.

Five of the eight patients in Group A used a transdermal preparation that contained exclusively Lidocaine and Ketamine, although the percentages of each of these components that were prescribed varied from patient to patient. One patient out of these five who were prescribed and exclusively 
used Lidocaine/Ketamine in the transdermal preparation found the cream effective.

The amount of Lidocaine/Ketamine in the one patient who did not find the transdermal effective in Group A was the lowest (Lidocaine 3\%/Ketamine 5\%) when compared with the preparations used in those patients who found the transdermal cream effective. The clinical history for this patient stated that the patient "is happy with pain control, though still rates it a 7/10". We assumed the pain was originally ranked 7/10, though the previous clinical notes did not state this, categorizing the effect of the cream as "nonmeaningful relief". Despite the unchanged pain ranking, the same clinical note also stated there was "resolution of a 'snake' like rippling sensation in...abdomen," so there was, in fact, complete resolution of one component of this patient's neuropathic pain.

It is possible the transdermal cream was not as effective in Group $\mathrm{C}$ because patients in those groups did not have a definitive diagnosis of neuropathic pain. In addition, Group C contained only three patients.

\section{Follow-up}

Patients in Groups A and C had clinic notes describing follow-up after use of the transdermal preparation, however, subsequent clinic notes do not mention if there was continued use of the transdermals and their effectiveness. It was, therefore, difficult to determine duration of use. For these patients, as well as the patients in Groups B and D, it is possible other primary complaints dominated the clinic visit and the neuropathic pain was either not as significant or had resolved, as many of the follow-up clinic notes simply did not mention "neuropathic pain" or "transdermal cream".

\section{Adverse Reactions}

Adverse effects were not life-threatening and were limited to the dermatologic system. It was difficult to determine which components of the transdermal preparation may have caused a reaction. The patient who developed hives had applied a $2 \%$ Lidocaine $/ 5 \%$ Ketamine/10\% Diclofenac in PLO base. Despite the hives, this patient "found the cream helpful, with a numbing effect" and had "fair amount of initial improvement" with this formulation. A different compound of $2 \%$ Lidocaine $/ 10 \%$ Diclofenac in a Lidoderm base was prescribed instead and upon follow-up, the patient "didn't notice it to be as effective, but did not have the dermatitis". As two changes to the prescription were made, it is unclear if the reaction was due to the PLO base or the Ketamine in this patient. The decreased effectiveness with the second transdermal formulation was most likely related to exclusion of Ketamine.

The patient who developed dermatitis had applied a transdermal containing 10\% Lidocaine/10\% Ketamine (base unknown). It was discontinued and the patient was given a transdermal containing 10\% Lidocaine/5\% Diclofenac/5\% Amitriptyline (base unknown) that also produced dermatitis; we assume this was discontinued. Again, it was difficult to determine which component caused the reaction. There was no further mention of a new transdermal being prescribed, but the subsequent clinic note stated continued use of a
Lidocaine/Ketamine transdermal preparation (base unknown), but no prescription was found in the chart, and no further follow-up was documented regarding adverse effects, effectiveness of this preparation, or a refill of the prescription. Without knowing the compounding base of any of the transdermals or the percentages of Lidocaine/Ketamine this patient was prescribed, it is difficult to ascertain the reason for the dermatitis.

\section{Study Limitations}

The retrospective nature of this study standardization could not be ensured. For example, the transdermal could contain varying percentages of its components, not all components could be determined if copies of prescriptions were absent, doses of co-medications taken orally could not be kept stable, follow-up of effectiveness could not be ensured, and duration of use could not be quantified. It was difficult to ascertain if patients filled out their prescriptions for the transdermals, as multiple pharmacies are known to compound transdermal creams.

The sample size of convenience allowed for selection bias and limits the generalizability of the results.

Lastly, a number of charts did not contain the clinical information necessary for this study's raw data collection. As evidenced by the division of the patients into groups for this study's analysis, not all patient charts specifically stated if a patient was diagnosed with NeP. Additionally, percentages of the components in a transdermal cream prescribed for a number of patients included in this study were not specified in the clinic notes, nor did all charts contain a copy of the prescription for the transdermal cream. As mentioned above, follow-up clinic notes did not always address $\mathrm{NeP}$ or the effect of the previously prescribed transdermal cream.

In conclusion, this study found transdermal creams containing Ketamine and Lidocaine were effective in $73 \%$ of patients with neuropathic pain. Transdermal cream containing Ketamine and Lidocaine may be a good alternative to oral medications. Future directions in examining the effectiveness of transdermal Ketamine and Lidocaine for the management of NeP may involve a prospective study employing a transdermal cream containing only Ketamine and Lidocaine.

\section{CONFLICT OF INTEREST}

None declared.

\section{ACKNOWLEDGEMENT}

None declared.

\section{REFERENCES}

[1] Torrance N, Smith BH, Bennett MI, Lee AJ. The epidemiology of chronic pain of predominantly neuropathic origin. Results from a general population survey. J Pain 2006; 7: 281-9.

[2] Moulin DE, Clark AJ, Gilron, I, et al. Pharmacological management of chronic neuropathic pain - Consensus statement and guidelines from the Canadian Pain Society. Pain Res Manag 2007; 12: 13-21.

[3] Gilron I, Watson CPN, Cahill CM, Moulin DE. Neuropathic pain: a practical guide for the clinician. CMAJ 2006; 175: 265-75. 
[4] Vadaurri V. Topical treatment of neuropathic pain. Int J Pharm Compd 2008; 12: 182-90.

[5] Sawynok J. Topical and peripherally acting analgesics. Pharmacol Rev 2003; 55: 1-20.

[6] Jovey RD, ed. Managing pain: the Canadian healthcare professional's reference. Second ed. Toronto: Healthcare \& Financial Publishing 2002.

[7] Woolf CJ. Pain: Moving from symptom control toward mechanism-specific pharmacologic management. Ann Intern Med 2004; 140: 441-51.

[8] de Leon-Casasola OA. Multimodal approaches to the management of neuropathic pain: the role of topical analgesia. J Pain Symptom Manage 2007; 33: 356-64.

[9] Woolf CJ. Central sensitization: uncovering the relation between pain and plasticity. Anesthesiology 2007; 106: 864-7.

[10] Dworkin RH, O’Connor AB, Backonja M, et al. Pharmacologic management of neuropathic pain: evidence-based recommendations. Pain 2007; 132: 237-51.

[11] Ball AM, Smith KM. Optimizing transdermal drug therapy. Am J Health Syst Pharm 2008; 65:1337-46.
[12] McCleane, G. Topical application of doxepin hydrochloride, capsaicin and a combination of both produces analgesia in chronic human neuropathic pain: a randomized, double-blind, placebocontrolled study. Br J Clin Pharmacol 2000; 49: 574-9.

[13] Murdan, S. A review of pluronic lecithin organogel as a topical and transdermal drug delivery system. Hosp Pharm 2005; 12: 267-70.

[14] Sandoval JA, Furlan, AD, Mailis-Gagnon A. Oral methadone for noncancer pain. Clin J Pain 2005; 21: 503-12.

[15] Lynch ME, Clark AJ, Sawynok J, Sullivan MJL. Topical 2\% amitriptyline and $1 \%$ ketamine in neuropathic pain syndromes. Anesthesiology 2005; 103: 140-6.

[16] Ho K-Y, Huh BK, White WD, Yeh C-C, Miller EJ. Topical amitriptyline versus lidocaine in the treatment of neuropathic pain. Clin J Pain 2008; 24: 51-5.

[17] Meier T, Wasner G, Faust, M, et al. Efficacy of lidocaine patch 5\% in the treatment of focal peripheral neuropathic pain syndromes: a randomized, double-blind, placebo-controlled study. Pain 2003; 106: 151-8.

[18] Khaliq W, Alam S, Puri N. Topical lidocaine for the treatment of postherpetic neuralgia. Cochrane Database Syst Rev 2007, Issue 2. Art. No.: CD004846. DOI: 10.1002/14651858.CD004846.pub2.

(C) Tam and Furlan; Licensee Bentham Open.

This is an open access article licensed under the terms of the Creative Commons Attribution Non-Commercial License (http://creativecommons.org/licenses/by-nc/3.0/) which permits unrestricted, non-commercial use, distribution and reproduction in any medium, provided the work is properly cited. 